\title{
Technè
}

La science au service de l'histoire de l'art et de la préservation des biens culturels

43 | 2016

Une Europe de la recherche en sciences du patrimoine

\section{Répertoire des techniques d'examen et d'analyse des œuvres du patrimoine culturel citées dans ce numéro}

Index of examination and analysis techniques of cultural heritage artefacts mentionned in this issue

\section{OpenEdition}

Journals

Édition électronique

URL : http://journals.openedition.org/techne/568

DOI : $10.4000 /$ techne.568

ISSN : 2534-5168

Éditeur

C2RMF

Édition imprimée

Date de publication : 1 août 2016

Pagination : 7

ISBN : 978-2-7118-6338-9

ISSN : $1254-7867$

\section{Référence électronique}

"Répertoire des techniques d'examen et d'analyse des œuvres du patrimoine culturel citées dans ce numéro », Technè [En ligne], 43 | 2016, mis en ligne le 19 décembre 2019, consulté le 15 septembre 2020. URL : http://journals.openedition.org/techne/568

\section{(c) (i) $\odot$}

La revue Technè. La science au service de l'histoire de l'art et de la préservation des biens culturels est mise à disposition selon les termes de la Licence Creative Commons Attribution - Pas d'Utilisation Commerciale - Pas de Modification 4.0 International. 


\section{Répertoire des techniques d'examen et d'analyse des œuvres du patrimoine culturel citées dans ce numéro}

\section{Index of examination and analysis techniques of cultural heritage artefacts mentionned in this issue}

\section{Méthodes d'examen - Examination methods}

AFM (Atomic force microscopy) : Microscopie à force atomique.

DHSPI (Digital Holographic Speckle Pattern Interferometry) : Holographie numérique et interférométrie de Speckle.

FEG-SEM (Scanning electron microscopy with field emission gun) : Microscopie électronique à balayage à canon à émission de champ (MEB-FEG)*

High resolution digital photography : Photographie numérique en haute résolution

Infrared (IR) false colour photography : Photographie dans l'infrarouge (IR)*.

Infrared (IR) reflectography : Réflectographie infrarouge*.

Nuclear Magnetic Resonance Tomography (NMR Tomography) : Tomographie par résonance magnétique nucléaire (tomographie RMN).

OCT (Optical coherent tomography) : Tomographie optique cohérente.

Raking light photography : Photographie en lumière rasante*.

SEM (Scanning electron microscopy) : Microscopie électronique à balayage $(\mathrm{MEB})^{*}$.

$\mathrm{T}-\mathrm{Hz}$ (Terahertz imaging) : Imagerie térahertz.

Ultraviolet (UV) induced fluorescence photography: Photographie de fluorescence sous ultraviolet (UV)*

Visible light photography: Photographie en lumière visible*.

X-ray radiography : Radiographie X*.

Méthodes d'analyse par spectrométrie optique - Optical spectrometry analysis methods

FT-IR (Fourier Transform infrared reflectance spectroscopy) : Spectrométrie infrarouge par transformée de Fourier*.

FORS (Fibre optic reflectance spectroscopy) : Spectrométrie par réflexion à l'aide de fibre optique.

MNIR (Mid-near infrared reflectance spectroscopy) : Spectrométrie dans le proche et moyen infrarouge.
Raman (Raman spectroscopy) : Spectroscopie (ou spectrométrie) Raman*

TCSPC (Measurement of luminescence decay lifetime with time-correlated singlephoton counting) : Mesure de fluorescence par comptage de photon unique, corrélé en temps.

$\mathrm{UV}$-Vis (Ultraviolet-visible reflectance and fluorescence spectroscopy) Spectrométrie bimodale (réflexion et fluorescence) UV-Vis*.

Méthodes d'analyse par spectrométrie X $X$-ray spectrometry methods

EDXRD (Energy dispersive X-ray diffraction) : Diffraction X par dispersion en énergie.

SEM-EDXA (Scanning electron microscopy with energy dispersive X-ray spectrometry) : Micro-analyse X couplée au microscope électronique à balayage (MEB-EDX)*.

XRD (X-ray diffraction) : Diffraction $\mathrm{X}^{*}$

XRF (X-ray fluorescence analysis) : Analyse par fluorescence X (AFX)*.

Méthodes d'examen et d'analyse par rayonnement synchrotron - Synchrotron radiation (SR) induced examination and analytical techniques

SR-XRD (SR-X-ray diffraction) : Diffraction $\mathrm{X}$ par rayonnement synchrotron.

SR-XPS (SR-X-ray photoelectron spectroscopy) : Spectrométrie de photoélectrons induits par rayonnement synchrotron

XANES (X-ray absorption near edge structures) : Spectrométrie d'absorption des rayons $\mathrm{X}$ au voisinage des discontinuités.

XAS (X-ray absorption spectroscopy) : Spectrométrie d'absorption de rayons $\mathrm{X}$.

Méthodes d'analyse par faisceaux d'ions Ion beam analysis methods

ERDA (Elastic recoil detection analysis) : Analyse par détection de recul élastique*.

IBA (Ion beam analysis) : Analyse par faisceau d'ions*.
IBIL (Ion beam induced luminescence) : Luminescence induite par faisceau d'ions.

NRA (Nuclear reaction analysis) : Analyse par réactions nucléaires*.

PESA (Proton elastic scattering analysis) : Analyse par diffusion élastique de protons.

PIGE (Particle induced gamma-ray emission) : Analyse par émission de rayons gamma induits par particules*.

PIXE (Particle induced X-ray emission) : Analyse par émission de rayons X induits par particule*.

RBS (Rutherford backscattering spectrometry) : Spectrométrie de rétrodiffusion Rutherford*

STIM (Scanning transmission ion microscopy) : Microscopie à balayage par transmission d'ions.

Méthodes d'examen et d'analyse par

faisceaux de neutrons - Neutron induced examination and analytical techniques

NAA (Neutron activation analysis) : Analyse par activation neutronique*

ND (Neutron diffraction) : Diffraction neutronique.

Neutron induced prompt gamma tomography: Tomographie par gamma de capture induits par neutrons.

NR (Neutron radiography) : Neutronographie*.

NT (Neutron tomography) : Tomographie neutronique.

PGAA (Prompt gamma activation analysis) : Analyse par gamma de capture.

SANS (Small-angle neutron scattering) : Diffusion de neutrons aux petits angles.

TOF-ND (Time-of-flight neutron diffraction) : Diffraction neutronique par temps de vol.

Pour plus de détails, se reporter aux définitions et aux équivalents dans diverses langues européennes proposées par la base CAMEO

(http://cameo.mfa.org/wiki/Main_Page) For more details, one can refer to the definitions and the equivalent terms in various European languages proposed by CAMEO base.

Les techniques repérées par le symbole figurent dans la base CAMEO au 22/06/2016Techniques tracked with * are included in CAMEO base on June 22nd 2016. 